\title{
A Predictive Model of EGFR Mutation Status in Patients with Lung Adenocarcinoma Based on PET/CT Metabolic Indexes and Clinicopathological Variables
}

\section{Yanlong Yang}

Shantou Central Hospital

\section{Shuchen Shi}

Shantou Central Hospital

Qianzhun Huang

Maoming People's Hospital

Wenzhao Zhong

Guangdong Academy of Medical Sciences: Guangdong Provincial People's Hospital

Juntao Lin

Guangdong Academy of Medical Sciences: Guangdong Provincial People's Hospital

Weichao Huang

Shantou Central Hospital

Zizhe Li

Shantou Central Hospital

Bin Lan ( $\square$ stlanbin2020@163.com )

Shantou Central Hospital

\section{Research article}

Keywords: Lung adenocarcinoma, EGFR, PET/CT, standardized uptake value, predictive model

Posted Date: December 1st, 2020

DOl: https://doi.org/10.21203/rs.3.rs-115858/v1

License: (a) (1) This work is licensed under a Creative Commons Attribution 4.0 International License. Read Full License 


\section{Abstract}

Purpose

The purpose of this study was to create a mathematical model based on the metabolic parameters of PET/CT with clinicopathological characteristics to predict the EGFR mutation status of patients with lung adenocarcinoma.

Methods

This study retrospectively enrolled patients with lung adenocarcinoma who underwent surgical treatment at two centres in China between January 2012 and December 2015. PET/CT metabolic parameters and Classical EGFR mutation status detection by molecular pathology were performed before and after surgery, and we analysed the associations of EGFR mutation status with patient sex, age, smoking history, maximum primary lesion diameter, carcinoembryonic antigen (CEA), neuron-specific enolase (NSE), cytokeratin 19 fragment (CYFRA21-1), TNM stage and histopathological subtype of lung adenocarcinoma.

Results

A total of 310 patients were included, comprising 161 with EGFR mutations (51.9\%) and 149 with wild-type EGFR (48.1\%). EGFR mutations were more common in females, non-smokers, and those with stage IV disease, a low SUVmax, and $\leq 35 \mathrm{~mm}$ nodules, whereas wild-type EGFR was more common in males, smokers, and those with a solid growth pattern. Multivariate analysis suggested that liver SUVratio, smoking history, tumour size, TNM stage, and solid growth pattern can predict EGFR mutation status, and these factors were used to construct a mathematical model.

\section{Conclusion}

The prediction model constructed in this study based on clinicopathological characteristics and PET/CT parameters might offer a basis by which to predict Classical EGFR status and provide a certain reference value for guiding the use of EGFR-tyrosine kinase inhibitor (EGFR-TKI) treatment in patients with lung adenocarcinoma.

\section{Background}

According to statistics from the World Health Organization, in 2018, lung cancer was the most common malignant tumour (accounting for approximately $11.6 \%$ of all cancers) and ranked first in morbidity and mortality [1]. As molecular biology research has progressed, people have begun to discover molecular pathways that drive the occurrence of malignant tumours, the most prominent of which is epidermal growth factor receptor (EGFR) [2]. The 2014 PIONEER study showed that the EGFR mutation rate in patients with non-small cell lung cancer in China is approximately $50 \%$ [3]. A series of clinical studies have confirmed that EGFR-tyrosine kinase inhibitors (EGFRTKIs) have a significant effect on patients with EGFR-mutated non-small cell lung cancer and can significantly prolong the progression-free survival (PFS) of these patients. Subsequently, EGFR-TKIs have been recommended as the first-line treatment for patients with advanced lung cancer with EGFR mutations. Therefore, it is particularly important to clarify the EGFR mutation status of such patients. 
The exon 19 deletion mutation and the L858R replacement mutation in exon 21 are the most common types of EGFR mutations. Tissue biopsy is the gold standard for clarifying the patient's tissue subtype and molecular subtype, but it is invasive and limited by the lesion location, surgeon's operation technique, pathologist's level of discriminating small specimens, patient's physical condition score, and detection delays[4, 5].Therefore, it is imperative to find a simple and noninvasive method to predict the EGFR mutation status.

In recent years, noninvasive tumour metabolic imaging has been widely used in the diagnosis and treatment evaluation of malignant tumours. The use of positron emission tomography/computed tomography (PET/CT) in this context is based on the observation that glucose metabolism is increased in malignant tumour tissues- this can be exploited by using a ${ }^{18} \mathrm{~F}$-fluorodeoxyglucose $\left({ }^{18} \mathrm{~F}-\mathrm{FDG}\right)$ tracer to highlight tumour cells with high metabolism on the PET/CT image [6]. Moreover, patients with mutant EGFR show decreased expression of some proteins related to glucose metabolism, for example resulting in the downregulation of the expression of glucose transporter-1 (GLUT-1); this may cause patients with different EGFR statuses to show abnormal metabolic indicators on PET/CT $[7,8]$. This suggests that the metabolic index calculated from PET/CT may be used as a noninvasive biological marker to indicate EGFR mutation status. We will test this hypothesis later.

The purpose of this study was to analyse the relationships of PET/CT metabolic parameters and general clinicopathological characteristics with EGFR mutation status of patients with lung adenocarcinoma, and thereby to create a mathematical model that incorporates the predictive factors.

Lung squamous cell carcinoma has been found to be significantly different from lung adenocarcinoma in terms of its metabolic characteristics [9]. The SUVmax value of lung squamous cell carcinoma is significantly higher than that of lung adenocarcinoma, and the EGFR mutation rate in lung squamous cell carcinoma patients is extremely low, which may lead to a significant increase in the SUVmax of patients with wild-type EGFR. Therefore, all patients included in this study had lung adenocarcinoma.

\section{Methods}

2.1 Patients and inclusion criteria: This study retrospectively enrolled patients with lung cancer who underwent lobectomy and sublobectomy (including lung wedge resection and segmental resection) at the Department of Cardiothoracic Surgery of Shantou Central Hospital and the Lung Research Institute of Guangdong Provincial People's Hospital from January 1, 2012, to December 31, 2015. This article is a retrospective study, so the principle of patient informed consent is rejected. The inclusion criteria were as follows: (1) preoperative PET/CT examination; (2) lung infiltrating adenocarcinoma clearly identified by postoperative pathology; and (3) known EGFR mutation status. The exclusion criteria were as follows: (1) primary lesion diameter $<1 \mathrm{~cm}$ or primary lesions with ground-glass opacity (GGO); (2) any previous antitumour treatment before PET/CT; (3) history of previous malignant tumours; (4) multiple primary lung cancers; and (5) diabetes with a blood sugar level not controlled within the normal range. In recruited patients, we collected patient-related clinical data and PET/CT imaging data, including sex, age, smoking status, carcinoembryonic antigen (CEA), neuron-specific enolase (NSE), cytokeratin 19 fragment (CYFRA21-1), TNM stage, maximum tumour diameter, maximum standardized uptake value (SUVmax) of the primary lesion, mediastinal SUVratio, and liver SUVratio. Tumour staging was based on the International association for the Study of Lung Cancer (IASLC) 8th edition TNM lung cancer staging system.

2.2 ${ }^{18}$ F-FDG PET/CT imaging method and image analysis: The patients underwent PET/CT imaging within 1 month before surgery. For PET/CT, the scan was performed using a Sensation Biograph Somatom 16 PET/CT 
scanner (Siemens Medical Solutions, Germany). Patients were required to fast for more than $4 \mathrm{~h}$ prior to the scan, and blood glucose levels were required to be $<8 \mathrm{mmol} / \mathrm{L}$ before receiving the injection of ${ }^{18} \mathrm{~F}-\mathrm{FDG}$. After an intravenous injection of ${ }^{18} \mathrm{~F}-\mathrm{FDG}(5.92 \mathrm{MBq} / \mathrm{kg})$ for an hour, PET/CT images were acquired from the base of the skull to the middle of the thigh. CT scans (voltage $120 \mathrm{kV}, 50 \mathrm{mAs}$ ) for attenuation correction and anatomic localization were acquired from the upper thigh to the base of the skull. Contrast-enhanced CT (CE-CT) scans were conducted in the same position after the completion of the PET scan (120 kV, $140 \mathrm{mAs})$. Two senior nuclear medicine physicians from Department of PET Center with $>6$ years of experience interpreted the PET/CT images.

On the PET image, the regions of the lungs with abnormally high metabolism were used to outline the region of interest (ROI), and SUVmax = average ROI activity $(\mathrm{MBq} / \mathrm{ml}) /$ injected dose $[\mathrm{MBq} / \mathrm{body}$ weight $(\mathrm{g})]$. If there were areas with no abnormally high metabolism on the image, the area corresponding to the lesion on the CT image was selected as the ROI. The mediastinal blood pool SUVmax was measured in the main pulmonary artery, and the hepatic blood pool SUVmax was measured in the parenchymal part of the liver. The ratio of the SUVmax to the blood pool was used to control for technical differences between scans. SUVratio = SUVmax of the primary tumour ROI/SUVmax of the mediastinal blood pool (or liver blood pool).

2.3 Histopathology and EGFR mutation status detection: Classical EGFR mutation include the deletion of exon 19 (19del) and the point mutation of L858R in exon 21, accounting for more than $85 \%$ of the entire EGFR mutation, and these patients respond well to TKI drugs. Therefore, our study only included patients with classical EGFR mutation. If classical EGFR mutation was detected, the tumour was identified as an EGFR mutant, otherwise, the tumour was identified as EGFR wild type. The surgical specimens were evaluated by two senior pathologists with \6 years of experience and were clearly diagnosed as infiltrating lung adenocarcinoma. According to the international multidisciplinary classification standard of lung adenocarcinoma established by the IASLC, American Thoracic Society (ATS) and European Respiratory Society (ERS) in 2011, the invasive adenocarcinoma subtypes were classified as follows: 1 . adherent growth pattern (nonmucinous bronchioloalveolar carcinoma (BAC) in the original classification standard, and diameter $\geq 5 \mathrm{~mm}$ ); 2 . acinar-like growth pattern; 3. papillary growth pattern; 4. micropapillary shape growth pattern; 5 . solid growth pattern; and 6 . variants of lung adenocarcinoma (including mucinous adenocarcinoma, colloid carcinoma, intestinal adenocarcinoma, and foetal adenocarcinoma). EGFR mutation status was detected using the amplification refractory mutation system (ARMS) method, after specific primers and probes are combined with the DNA template, Taq DNA polymerase uses deoxynucleotide (dNTP) as the substrate. The internal reference gene and the mutant gene in specific regions of exons 19 and 21 of the EGFR gene were amplified in vitro. The fluorescence is released by hydrolysis of specific probes, and the progress of the PCR reaction is monitored to determine the EGFR gene mutation.

2.4 Statistical methods: All data were statistically analysed using SPSS 21.0 and MedCalc 18.9.1 software. An independent sample t-test was used to compare continuous variables between EGFR mutant and EGFR wild type, including primary lesion SUVmax, age, and maximum tumour diameter. Continuous variables are reported as the mean \pm standard deviation. The Mann-Whitney $U$ test was used to compare nonnormally distributed continuous variables, including CEA, NSE, and CYFRA21-1. The chi-square test and Fisher's exact test were used to compare categorical variables, including sex, smoking status, TNM stage, and pathological subtype. The assignment of categorical variables is shown in Table 1. Receiver operating characteristic (ROC) curves were created for variables with $\mathrm{P}<0.1$ in univariate analysis, and the intercept values of the continuous variables were obtained. In multivariate analysis, the variables with $\mathrm{P}<0.1$ were included in the binary logistic regression (LR) analysis, and the forward LR and backward LR distributions were used to identify effective variables for predicting EGFR 
mutation status. Based on the results of the binary logistic regression analysis, a mathematical model for predicting EGFR mutation was created. A ROC curve was created, and the area under the curve (AUC) was calculated to evaluate the predictive value of the model. $P \leq 0.05$ indicates statistical significance.

Table 1 Assignment table of categorical variables.

\begin{tabular}{|c|c|}
\hline $\begin{array}{l}\text { Categorical } \\
\text { variables }\end{array}$ & Assignment method \\
\hline Sex & Male $=1$, female $=0$ \\
\hline Smoking history & Smoker $=1$, Non-smoker $=0$ \\
\hline $\begin{array}{l}\text { Pathological } \\
\text { adenocarcinoma } \\
\text { subtype }\end{array}$ & $\begin{array}{l}\text { Adherent growth pattern }=1 \text {, Acinar-like growth pattern }=2 \text {, Papillary growth pattern }=3 \text {, } \\
\text { Micropapillary shape growth pattern }=4 \text {, Solid growth pattern }=5 \text {, Variants of lung } \\
\text { adenocarcinoma }=6\end{array}$ \\
\hline TNM staging & Stage $I=1$, Stage $I I=2$, Stage $I I I=3 \rrbracket$ Stage $I V=4$ \\
\hline $\begin{array}{l}\text { EGFR mutation } \\
\text { status }\end{array}$ & EGFR mutant $=1 \rrbracket$ EGFR wild type $=0$ \\
\hline $\begin{array}{l}\text { EGFR mutation } \\
\text { site }\end{array}$ & EGFR wild type $=0$ QEGFR19 exon mutations $=1 \otimes$ EGFR21 exon mutations $=2$ \\
\hline
\end{tabular}

\section{Results}

3.1 Analysis of EGFR mutation status: Of the 310 patients enrolled, 149 (48.1\%) had wild-type EGFR; of the remaining 161 (51.9\%) with EGFR mutations, 84 (52.2\%) had EGFR21 exon mutations, and 65 (40.4\%) had EGFR19 exon mutations. The associations of categorical and numerical clinical variables with EFGR mutation status are shown in Tables 2 and 3 , respectively. The EGFR mutation rate was higher in female patients than in male patients $(65.2 \%$ vs. $40.8 \%$, odds ratio $(O R)=2.721, P=0.000)$ and higher in non-smokers than in smokers ( $65.3 \%$ vs. $30.8 \%, O R=4.215, P=0.000)$. The ROC curve shows that the cutoff point of the largest tumour diameter in each patient was $35 \mathrm{~mm}$, and the calculated AUC was 0.629 (95\% confidence interval (Cl), 0.573-0.683). The EGFR mutation rate in patients in which the largest tumour diameter $\leq 35 \mathrm{~mm}$ was greater than that in patients with largest tumour diameter $>35 \mathrm{~mm}(32.7 \%$ vs. $61.2 \%$, OR value $=0.307, \mathrm{P}=0.000)$. There was no statistically significant difference in age according to presence of EGFR mutation ( $P=0.532)$. The EGFR mutation rates among TNM stage I, II, III, and IV tumours was no significant difference ( $P=0.068$ ), which were $55.2 \%, 44.3 \%, 44.6 \%$, and $73.7 \%$, respectively. But there was a significant difference between stages II and IV ( $P=0.025)$. The EGFR mutation rates of the acinar-like growth pattern and papillary-like growth pattern did not significantly differ, but were higher than that of the solid growth pattern $(P=0.000)$. All variants of invasive adenocarcinoma had wild-type EGFR.

Table 2 Associations of categorical clinical variables and SUVmax with EGFR mutation status. 


\begin{tabular}{|c|c|c|c|c|c|c|c|}
\hline \multirow[t]{2}{*}{ Variable } & \multirow{2}{*}{$\begin{array}{l}\text { Mutated } \\
\text { EGFR,n (\%) }\end{array}$} & \multirow{2}{*}{$\begin{array}{l}\text { Wild-type } \\
\text { EGFR,n (\%) }\end{array}$} & \multirow{2}{*}{$\begin{array}{l}\mathrm{P} \\
\text { value }\end{array}$} & \multirow[t]{2}{*}{$\square^{2}$} & \multirow{2}{*}{$\begin{array}{l}\mathrm{OR} \\
\text { value }\end{array}$} & \multicolumn{2}{|l|}{$95 \% \mathrm{Cl}$} \\
\hline & & & & & & $\begin{array}{l}\text { Lower } \\
\text { limit }\end{array}$ & $\begin{array}{l}\text { Upper } \\
\text { limit }\end{array}$ \\
\hline Sex & & & 0.000 & 18.363 & 2.721 & 1.713 & 4.323 \\
\hline Female & $92(65.2)$ & $49(34.8)$ & & & & & \\
\hline Male & $69(40.8)$ & $100(59.2)$ & & & & & \\
\hline Smoking history & & & 0.000 & 34.926 & 4.215 & 2.584 & 6.874 \\
\hline No & $124(65.3)$ & $66(34.7)$ & & & & & \\
\hline Yes & $37(30.8)$ & $83(69.2)$ & & & & & \\
\hline TNM staging & & & 0.068 & 7.118 & & & \\
\hline I & $91(55.2)$ & $74(44.8)$ & & & & & \\
\hline II & $27(44.3)$ & $34(55.7)$ & & & & & \\
\hline III & $29(44.6)$ & $36(55.4)$ & & & & & \\
\hline IV & $14(73.7)$ & $5(26.3)$ & & & & & \\
\hline $\begin{array}{l}\text { Maximum primary tumour } \\
\text { diameter }(\mathrm{mm})\end{array}$ & & & 0.000 & 22.267 & 0.307 & 0.186 & 0.506 \\
\hline$>35$ & $33(32.7)$ & $68(67.3)$ & & & & & \\
\hline$\leq 35$ & $128(61.2)$ & $81(38.8)$ & & & & & \\
\hline CEA $(\mu \mathrm{g} / \mathrm{l})$ & & & 0.000 & 20.032 & 0.332 & 0.203 & 0.542 \\
\hline$>2.53$ & $75(40.5)$ & $110(59.5)$ & & & & & \\
\hline$\leq 2.53$ & $76(67.3)$ & $37(32.7)$ & & & & & \\
\hline NSE $(\mu \mathrm{g} / \mathrm{l})$ & & & 0.017 & 5.686 & 0.564 & 0.352 & 0.905 \\
\hline$>16.09$ & $50(41.7)$ & $70(58.3)$ & & & & & \\
\hline$\leq 16.09$ & $95(55.9)$ & $75(44.1)$ & & & & & \\
\hline CYFRA21-1 $(\mu \mathrm{g} / \mathrm{l})$ & & & 0.038 & 0.608 & 0.608 & 0.379 & 0.974 \\
\hline$>2.55$ & $75(44.6)$ & $93(55.4)$ & & & & & \\
\hline$\leq 2.55$ & $69(57)$ & $52(43)$ & & & & & \\
\hline $\begin{array}{l}\text { Pathological } \\
\text { adenocarcinoma subtype }\end{array}$ & & & 0.000 & 44.786 & & & \\
\hline Adherent growth pattern & $6(66.7)$ & $3(33.3)$ & & & & & \\
\hline Acinar-like growth pattern & $127(62.6)$ & $76(37.4)$ & & & & & \\
\hline Papillary growth pattern & $10(66.7)$ & $5(33.3)$ & & & & & \\
\hline Micropapillary shape growth & $1(50)$ & $1(50)$ & & & & & \\
\hline
\end{tabular}




\begin{tabular}{|llllllll|}
\hline pattern & & & & & & \\
\hline $\begin{array}{l}\text { Variants of lung } \\
\text { adenocarcinoma }\end{array}$ & $0(0)$ & $9(100)$ & & & & & \\
\hline Primary SUVmax & & & 0.000 & 19.737 & 0.345 & 0.214 & 0.555 \\
\hline 5.3 & $78(41.7)$ & $109(58.3)$ & & & & & \\
\hline$\leq 5.3$ & $83(67.5)$ & $40(32.5)$ & & & & & \\
\hline Mediastinal SUVratio & & & 0.000 & 12.818 & 0.415 & 0.255 & 0.674 \\
\hline$>3.1$ & $59(40.7)$ & $86(59.3)$ & & & & & \\
\hline$\leq 3.1$ & $81(62.3)$ & $49(37.7)$ & & & & & \\
\hline Liver SUVratio & & & 0.000 & 24.124 & 0.196 & 0.098 & 0.392 \\
$>4.1$ & $12(21.8)$ & $44(78.2)$ & & & & & \\
\hline$\leq 4.1$ & $128(58.2)$ & $92(41.8)$ & & & & & \\
\hline
\end{tabular}

Next, the factors related to EGFR19 exon mutations and EGFR21 exon mutations were analysed. For EGFR19 exon mutations, there were no statistically significant differences in the mutation rates between sexes or between smokers and non-smokers. The mutation rate of the EGFR19 exon was significantly different between tumours with a maximum primary lesion diameter $\leq$ or $>35 \mathrm{~mm}(25.4 \%$ vs. $11.9 \%, \mathrm{P}=0.006)$ or between primary lesions with an SUVmax $\leq 5.3$ or $>5.3(24.4 \%$ vs. $18.7 \%, P=0.23)$. There was no significant difference in the EGFR19 exon mutation rate according to TNM stage. For EGFR21 exon mutations, the mutation rates significantly differed between sexes ( $18.9 \%$ vs. $36.9 \%, P=0.000)$, and smokers had a significantly higher EGFR21 exon mutation rate than non-smokers ( $15 \%$ vs. $34.7 \%, P=0.000)$. The mutation rate of the EGFR21 exon was significantly different between tumours with a maximum primary lesion diameter $\leq 35 \mathrm{~mm}$ or $>35 \mathrm{~mm}(32.5 \%$ vs. $15.8 \%, \mathrm{P}=0.002)$ and between primary lesions with an SUVmax $\leq 5.3$ or $>5.3(32.2 \%$ vs. $19.8 \%, P=0.000)$. There was no significant difference in the EGFR21 exon mutation rate according to TNM stage.

Table 3 Associations of numerical clinical variables with EGFR mutation status. 


\begin{tabular}{|c|c|c|c|c|c|}
\hline Variable & Mutated EGFR & Wild-type EGFR & $\begin{array}{l}\mathrm{t} \\
\text { value }\end{array}$ & $\begin{array}{l}Z \\
\text { value }\end{array}$ & $\begin{array}{l}\mathrm{P} \\
\text { value }\end{array}$ \\
\hline Age & $61.18 \pm 9.32$ & $60.47 \pm 10.7$ & 0.626 & - & 0.532 \\
\hline $\begin{array}{l}\text { Primary tumour SUVmax, } \\
\text { mean } \pm \text { sd }\end{array}$ & $8.18 \pm 4.32$ & $5.88 \pm 3.41$ & 5.162 & - & 0.000 \\
\hline Mediastinal SUVratio, mean \pm sd & $4.54 \pm 2.64$ & $3.23 \pm 2$ & 4.657 & - & 0.000 \\
\hline Liver SUVratio, mean \pm sd & $3.28 \pm 2$ & $2.28 \pm 3.14$ & 4.890 & - & 0.000 \\
\hline $\begin{array}{l}\text { Maximum } \\
\text { primary tumour diameter, } \\
\text { mean } \pm \text { sd }\end{array}$ & $35.19 \pm 16.136$ & $27.83 \pm 10.011$ & 4.778 & - & 0.000 \\
\hline CEA $(\mu \mathrm{g} / \mathrm{l})$, median & $\begin{array}{l}4.49 \\
318.6)\end{array}$ & $\begin{array}{l}2.53 \\
206.95)\end{array} \mathbb{0 . 4 -}$ & - & -3.456 & 0.001 \\
\hline NSE $(\mu \mathrm{g} / \mathrm{l})$, median & $\begin{array}{l}15.85 \\
46.76)\end{array}$ & $\begin{array}{l}15.09 \\
58.48)\end{array} \quad \nabla 9.68-$ & - & -1.677 & 0.094 \\
\hline CYFRA21-1 $(\mu \mathrm{g} / \mathrm{l})$, median & $2.86 \quad 0.79-24.6)$ & $2.62 \quad 1.03-10.78)$ & - & -2.079 & 0.038 \\
\hline
\end{tabular}

3.2 Relationship between PET/CT metabolic parameters and EGFR mutation status: The primary lesion SUVmax, mediastinal SUVratio, and liver SUVratio were significantly lower in patients with EGFR mutations than in those with wild-type EGFR (all $P=0.000$; Table 2). Specifically, the EGFR mutation rate in patients with an SUVmax $\leq 5.3$ was higher than that in patients with an SUVmax $>5.3(61.5 \%$ vs $41.7 \%$, OR value $=0.345)$. The mutation rate in patients with a mediastinum SUVratio $\leq 3.1$ was higher than that in patients with mediastinal SUVratio $>3.1$ $(62.3 \%$ vs $40.7 \%$, OR value $=0.415)$. The mutation rate in patients with a liver SUVratio $\leq 4.1$ was higher than that in patients with a liver SUVratio $>4.1(58.2 \%$ vs $21.4 \%$, OR value $=0.196)$. There were no significant differences in the AUCs of SUVmax, mediastinal SUVratio, and liver SUVratio according to presence of EGFR mutation (Figure 1a).

3.3 Multivariate analysis to identify predictors of EGFR mutation status: Multivariate analysis showed that no history of smoking, maximum primary lesion diameter $\leq 35 \mathrm{~mm}$, pathological adenocarcinoma subtype (including adherent growth pattern, acinar-like growth pattern and papillary-like growth pattern), TNM stage IV, and liver SUVratio $\leq 4.1$ were significant predictors of Classical EGFR mutation (Table 4).

Table 4 Multivariate analysis of clinical data, PET/CT metabolic parameters, pathological subtypes of adenocarcinoma, and EGFR mutation status. 


\begin{tabular}{|c|c|c|c|c|c|}
\hline \multirow[t]{2}{*}{ Variable } & \multirow{2}{*}{$\begin{array}{l}\text { Regression } \\
\text { coefficient }\end{array}$} & \multirow[t]{2}{*}{ OR } & \multicolumn{2}{|l|}{$95 \% \mathrm{Cl}$} & \multirow[t]{2}{*}{$P$ value } \\
\hline & & & $\begin{array}{l}\text { Lower } \\
\text { limit }\end{array}$ & $\begin{array}{l}\text { Upper } \\
\text { limit }\end{array}$ & \\
\hline Smoking history & -1.22939 & 0.2727 & 0.1455 & 0.5109 & $<0.0001$ \\
\hline $\begin{array}{l}\text { Maximum } \\
\text { primary tumour diameter } \leq 35 \mathrm{~mm}\end{array}$ & 1.06846 & 2.9109 & 1.3874 & 6.1074 & 0.0047 \\
\hline \multicolumn{6}{|l|}{$\begin{array}{l}\text { Pathological adenocarcinoma } \\
\text { subtype }\end{array}$} \\
\hline Acinar-like growth pattern & 1.82264 & 6.1882 & 2.8017 & 13.668 & $<0.0001$ \\
\hline Papillary-like growth pattern & 2.21125 & 9.1271 & 1.9684 & 42.3197 & 0.0047 \\
\hline \multicolumn{6}{|l|}{ TNM stage } \\
\hline IV & 2.37914 & 10.7957 & 2.243 & 51.9593 & 0.003 \\
\hline Liver SUVratio $\leq 4.1$ & 1.30669 & 3.6939 & 1.4604 & 9.3438 & 0.0058 \\
\hline Constant & -2.85978 & & & & $<0.0001$ \\
\hline
\end{tabular}

\subsection{Mathematical model:}

Based on the results of the multivariate analysis, the following formula was used to establish the mathematical model for predicting the probably of the presence of EGFR mutation: $\mathrm{P}=\mathrm{e}^{\mathrm{x}} /\left(1+\mathrm{e}^{\mathrm{x}}\right), \mathrm{Q}=-2.85978-(1.22939 \times$ smoking history $)+(1.06846 \times$ diameter $)+(1.82264 \times$ acinar growth pattern $)+(2.21125 \times$ papillary growth pattern $)+(2.37914 \times$ stage IV $)+(1.30669 \times$ liver SUVratio), where e is the natural logarithm. Primary tumour diameter $\leq 35 \mathrm{~mm}$ means 1, otherwise means 0 . Liver SUVratio $\leq 4.1$ means 1 , otherwise means 0 .

ROC curves of one factor (smoking history), two factors (smoking history + liver SUVratio), three factors (smoking history + liver SUVratio + adenocarcinoma pathological subtype), four factors (smoking history + liver SUVratio + adenocarcinoma pathological subtype + maximum diameter of primary lesion), and five factors (smoking history + liver SUVratio + adenocarcinoma pathological subtype + primary lesion maximum diameter + TNM stage) for predicting EGFR mutation status were created, and the AUCs were compared (Figure $1 \mathrm{~b}$ and Table 5).

Table 5 Fitting degree of the mathematical model for predicting EGFR mutation status.

\begin{tabular}{|llllllllll|}
\hline & AUC & Sensitivity & Specificity & $95 \% \mathrm{Cl}$ & \multicolumn{3}{c|}{$\begin{array}{l}\text { Goodness-of-fit } \\
\text { test }\end{array}$} & Cox\&Snell & Negelkerke \\
& & & & $\begin{array}{l}\text { Lower } \\
\text { limit }\end{array}$ & $\begin{array}{l}\text { Upper } \\
\text { limit }\end{array}$ & $\square^{2}$ & $\begin{array}{l}\mathrm{P} \\
\text { value }\end{array}$ & $\mathrm{R}^{2}$ & $\mathrm{R}^{2}$ \\
\hline $\begin{array}{l}5 \\
\text { factors }\end{array}$ & 0.82 & 62.1 & 82.64 & 0.766 & 0.866 & 5.8165 & 0.4441 & 0.3087 & 0.4116 \\
\hline
\end{tabular}

\section{Discussion}


EGFR-TKIs have a significant effect on patients with EGFR-mutated non-small cell lung cancer and can significantly prolong progression-free survival (PFS) in patients [10]. As such, EGFR-TKIs have been used in firstline treatment in advanced non-small cell lung cancer patients with positive EGFR mutations. Therefore, to guide treatment decisions, it is imperative to find a simple and non-invasive method to predict the mutation status of EGFR. This study retrospectively analysed the association between PET/CT metabolic parameters and EGFR mutation status in patients with lung adenocarcinoma, identified that liver SUVmax ratio, adenocarcinoma subtype, tumour diameter, TNM stage and smoking history were associated with EGFR mutation status, and created a mathematical model based on these factors.

Our results confirmed that the primary tumour SUVmax, mediastinal SUVratio, and liver SUVratio were all negatively correlated with the EGFR mutation rate. Several previous studies have discussed the association between SUVmax and EGFR mutations, but the results are quite different. Among them, 8 studies showed that low SUVmax measurements can indicate EGFR mutations, similar to the results of this study $[7,11,12,13-17]$. Conversely, two studies pointed out that high SUVmax measurements suggest EGFR mutations $[18,19]$. Four further studies showed that the SUVmax is not associated with EGFR mutation status $[20,21,22,23]$. These differences may be because SUVmax is a semiquantitative indicator that can vary with FDG dose, blood glucose level, PET/CT scan time, fasting time, and ROI parameters. Therefore, SUVratio is added in this study to reduced errors.

Studies have shown that the lung adenocarcinoma subtype may be used as a predictor of EGFR mutations to guide EGFR-TKI treatment. Correspondingly, our multivariate regression analysis showed that the adenocarcinoma subtype and EGFR mutation status were significantly related, and we found that EGFR mutations were more common in lesions with adherent growth, acinar growth, and papillary growth patterns. Solid growth was the most common growth pattern in wild-type EGFR lesions, and invasive adenocarcinoma rarely exhibited EGFR mutations, which is basically consistent with another study [24]. Unlike that study, however, only solid nodules with a tumour diameter $\geq 10 \mathrm{~mm}$ were included in this study, and in the enrolment process, the main adenocarcinoma subtype on CT was ground-glass nodules. However, GGO lesions have poor glucose uptake and often show false-negative results on ${ }^{18}$ F-FDG PET/CT examinations, so patients with GGO lesions were not included in this study.

Lesion size was also an important factor. In this study, all patients had solid lung nodules $\geq 10 \mathrm{~mm}$. The statistical analysis of the ROC curve showed that a maximum tumour diameter of $35 \mathrm{~mm}$ was the cutoff point, and tumours with smaller diameters $(\leq 35 \mathrm{~mm}$ ) had a higher EGFR mutation rate than those with larger tumours, which is consistent with previous findings [25]. The results of this study suggest that patients with stage IV disease according to the TNM staging guidelines have a higher mutation rate, which is inconsistent with previous research [26], but the number of cases was small, and there may have been selection bias. Finally, univariate analysis showed that the EGFR mutation rate in non-smokers was higher than that in smokers $₫$ which is consistent with another study[27,28].

Compared with the prediction model that appeared in the previous study, the model created in this study added Liver SUVratio and mediastinal SUVratio for comparison to eliminate errors caused by factors such as ${ }^{18} \mathrm{~F}-\mathrm{FDG}$ injection dose, patient's blood glucose level, scan time and other factors during scanning. Sensitivity and specificity reached 62.1 and 82.64 respectively. 
Different EGFR mutation types may affect downstream signalling pathways in different ways, leading to different responses to EGFR-TKI treatment [29]. In this study, exon 19 and exon 21 mutations occurred in basically equal proportions, and the difference in SUVmax was nonsignificant. However, we found that clinical factors have different predictive values for EGFR19 exon mutations and EGFR21 exon mutations. Specifically, sex and smoking status failed to predict the mutation status of the EGFR19 exon but predicted significant differences in that of the EGFR21 exon. Patients with different EGFR mutation sites also had different clinicopathological characteristics: female patients and non-smokers often had EGFR21 exon mutations, and small nodules were often associated with EGFR19 exon mutations.

\section{Conclusion}

In summary, we created a model based on five factors: smoking history, tumour diameter, TNM staging, adenocarcinoma subtype and liver SUVratio, which can effectively predict the status of EGFR mutations in patients with lung adenocarcinoma. It shows the feasibility of using imaging and clinical pathological data to predict the classic EGFR mutation status of patients, and provides clinical reference for guiding treatment decisions.

\section{Abbreviations}

EGFR: epidermal growth factor receptor.

PET/CT: Positron Emission Computed Tomography.

SUV『Standardized Uptake Value.

${ }^{18}$ F-FDG: 18F-flurodeoxyglucose.

GGO :ground-glass opacity.

ROI :region of interest.

PCR: polymerase chain reaction.

ROC: Receiver operating characteristic.

AUC: Area Under Curve.

\section{Declarations}

- Ethics approval and consent to participate: Written informed consent of tumor acquisition for research has been obtained before surgery and approved by internal review board from Guangdong Lung Cancer Institute and Guangdong Provincial People's Hospital (Guangzhou, China, IRB approved protocol number GDREC2016175H). All procedures performed in studies involving human participants were in accordance with the ethical standards of the institutional, national research committee, 1964 Helsinki declaration and its later amendments or comparable ethical standards. This article does not contain any studies with animals performed by any of the authors. 
- Consent for publication: Not applicable.

- Availability of data and materials: The datasets used and/or analysed during the current study are available from the corresponding author on reasonable request.

- Competing interests: The authors declare that they have no competing interests.

- Funding:This research did not receive any specific grant from funding agencies in the public, commercial, or not-for-profit sectors.

- Authors' contributions: YYL, SSC: drafting/revising the manuscript, analysis or interpretation of data, statistical analysis.

HQZ, ZWZ, LJT, HWC, LZZ: revising the manuscript, analysis or interpretation of data.LB: acquisition of data, revising the manuscript, study concept or design, study supervision.

- All authors read and approved the final manuscript.

- Acknowledgements: Not applicable

\section{References}

1. Bray F, Ferlay J, Soerjomataram I, Siegel RL, Torre LA, Jemal A: Global cancer statistics 2018: GLOBOCAN estimates of incidence and mortality worldwide for 36 cancers in 185 countries. CA Cancer J Clin 2018, 68(6):394-424.

2. Yang L, Ying S, Hu S, Zhao X, Li M, Chen M, Zhu Y, Song P, Zhu L, Jiang T et al: EGFR TKIs impair lysosomedependent degradation of SQSTM1 to compromise the effectiveness in lung cancer. Signal Transduct Target Ther 2019, 4(1):25.

3. Shi Y, Au JS, Thongprasert S, Srinivasan S, Tsai CM, Khoa MT, Heeroma K, Itoh Y, Cornelio G, Yang PC: A prospective, molecular epidemiology study of EGFR mutations in Asian patients with advanced non-smallcell lung cancer of adenocarcinoma histology (PIONEER). J Thorac Oncol 2014, 9(2):154-162.

4. Sequist LV, Engelman JA, Lynch TJ: Toward noninvasive genomic screening of lung cancer patients. J Clin Oncol 2009, 27(16):2589-2591.

5. Chouaid C, Dujon C, Do P, Monnet I, Madroszyk A, Le Caer H, Auliac JB, Berard H, Thomas P, Lena H et al: Feasibility and clinical impact of re-biopsy in advanced non small-cell lung cancer: a prospective multicenter study in a real-world setting (GFPC study 12-01). Lung Cancer 2014, 86(2):170-173.

6. Plathow C, Weber WA: Tumor cell metabolism imaging. J Nucl Med 2008, 49 Suppl 2:43S-63S.

7. Takamochi K, Mogushi K, Kawaji H, Imashimizu K, Fukui M, Oh S, Itoh M, Hayashizaki Y, Ko W, Akeboshi M et al: Correlation of EGFR or KRAS mutation status with 18F-FDG uptake on PET-CT scan in lung adenocarcinoma. PLoS One 2017, 12(4):e0175622.

8. Chen L, Zhou Y, Tang X, Yang C, Tian Y, Xie R, Chen T, Yang J, Jing M, Chen F et al: EGFR mutation decreases FDG uptake in non-small cell lung cancer via the NOX4/ROS/GLUT1 axis. Int J Oncol 2019, 54(1):370-380.

9. de Geus-Oei LF, van Krieken JH, Aliredjo RP, Krabbe PF, Frielink C, Verhagen AF, Boerman OC, Oyen WJ: Biological correlates of FDG uptake in non-small cell lung cancer. Lung Cancer 2007, 55(1):79-87.

10. Minguet J, Smith KH, Bramlage P: Targeted therapies for treatment of non-small cell lung cancer-Recent advances and future perspectives. Int J Cancer 2016, 138(11):2549-2561. 
11. Na II, Byun BH, Kim KM, Cheon GJ, Choe dH, Koh JS, Lee DY, Ryoo BY, Baek H, Lim SM et al: 18F-FDG uptake and EGFR mutations in patients with non-small cell lung cancer: a single-institution retrospective analysis. Lung Cancer 2010, 67(1):76-80.

12. Mak RH, Digumarthy SR, Muzikansky A, Engelman JA, Shepard JA, Choi NC, Sequist LV: Role of 18Ffluorodeoxyglucose positron emission tomography in predicting epidermal growth factor receptor mutations in non-small cell lung cancer. Oncologist 2011, 16(3):319-326.

13. Guan J, Xiao NJ, Chen M, Zhou WL, Zhang YW, Wang S, Dai YM, Li L, Zhang Y, Li QY et al: 18F-FDG uptake for prediction EGFR mutation status in non-small cell lung cancer. Medicine (Baltimore) 2016, 95(30):e4421.

14. Cho A, Hur J, Moon YW, Hong SR, Suh YJ, Kim YJ, Im DJ, Hong YJ, Lee HJ, Shim HS et al: Correlation between EGFR gene mutation, cytologic tumor markers, 18F-FDG uptake in non-small cell lung cancer. BMC Cancer 2016, 16:224.

15. Minamimoto R, Jamali M, Gevaert O, Echegaray S, Khuong A, Hoang CD, Shrager JB, Plevritis SK, Rubin DL, Leung AN et al: Prediction of EGFR and KRAS mutation in non-small cell lung cancer using quantitative. Oncotarget 2017, 8(32):52792-52801.

16. Liu A, Han A, Zhu H, Ma L, Huang Y, Li M, Jin F, Yang Q, Yu J: The role of metabolic tumor volume (MTV) measured by [18F] FDG PET/CT in predicting EGFR gene mutation status in non-small cell lung cancer. Oncotarget 2017, 8(20):33736-33744.

17. Lv Z, Fan J, Xu J, Wu F, Huang Q, Guo M, Liao T, Liu S, Lan X, Liao S et al: Value of ${ }^{18}$ F-FDG PET/CT for predicting EGFR mutations and positive ALK expression in patients with non-small cell lung cancer: a retrospective analysis of 849 Chinese patients. Eur J Nucl Med Mol Imaging 2018, 45(5):735-750.

18. Huang CT, Yen RF, Cheng MF, Hsu YC, Wei PF, Tsai YJ, Tsai MF, Shih JY, Yang CH, Yang PC: Correlation of F-18 fluorodeoxyglucose-positron emission tomography maximal standardized uptake value and EGFR mutations in advanced lung adenocarcinoma. Med Oncol 2010, 27(1):9-15.

19. Ko KH, Hsu HH, Huang TW, Gao HW, Shen DH, Chang WC, Hsu YC, Chang TH, Chu CM, Ho CL et al: Value of $1 \otimes F-F D G$ uptake on PET/CT and CEA level to predict epidermal growth factor receptor mutations in pulmonary adenocarcinoma. Eur J Nucl Med Mol Imaging 2014, 41(10):1889-1897.

20. Chung HW, Lee KY, Kim HJ, Kim WS, So Y: FDG PET/CT metabolic tumor volume and total lesion glycolysis predict prognosis in patients with advanced lung adenocarcinoma. J Cancer Res Clin Oncol 2014, 140(1):8998.

21. Caicedo C, Garcia-Velloso MJ, Lozano MD, Labiano T, Vigil Diaz C, Lopez-Picazo JM, Gurpide A, Zulueta JJ, Zulueta J, Richter Echevarria JA et al: Role of [1 $\left.{ }^{1} \triangle \mathrm{F}\right] \mathrm{FDG}$ PET in prediction of KRAS and EGFR mutation status in patients with advanced non-small-cell lung cancer. Eur J Nucl Med Mol Imaging 2014, 41(11):2058-2065.

22. Lee SM, Bae SK, Jung SJ, Kim CK: FDG uptake in non-small cell lung cancer is not an independent predictor of EGFR or KRAS mutation status: a retrospective analysis of 206 patients. Clin Nucl Med 2015, 40(12):950958.

23. Choi YJ, Cho BC, Jeong YH, Seo HJ, Kim HJ, Cho A, Lee JH, Yun M, Jeon TJ, Lee JD et al: Correlation between (18)f-fluorodeoxyglucose uptake and epidermal growth factor receptor mutations in advanced lung cancer. Nucl Med Mol Imaging 2012, 46(3):169-175.

24. Travis WD, Brambilla E, Noguchi M, Nicholson AG, Geisinger KR, Yatabe Y, Beer DG, Powell CA, Riely GJ, Van Schil PE et al: International association for the study of lung cancer/american thoracic society/european 
respiratory society international multidisciplinary classification of lung adenocarcinoma. J Thorac Oncol 2011, 6(2):244-285.

25. Tu W, Sun G, Fan L, Wang Y, Xia Y, Guan Y, Li Q, Zhang D, Liu S, Li Z: Radiomics signature: A potential and incremental predictor for EGFR mutation status in NSCLC patients, comparison with CT morphology. Lung Cancer 2019, 132:28-35.

26. Liu Q, Sun D, Li N, Kim J, Feng D, Huang G, Wang L, Song S: Predicting EGFR mutation subtypes in lung adenocarcinoma using. Transl Lung Cancer Res 2020, 9(3):549-562.

27. Yao G, Zhou Y, Gu Y, Wang Z, Yang M, Sun J, Luo Q, Zhao H: Value of combining PET/CT and clinicopathological features in predicting EGFR mutation in Lung Adenocarcinoma with Bone Metastasis. J Cancer 2020, 11(18):5511-5517.

28. Yang B, Ji HS, Zhou CS, Dong H, Ma L, Ge YQ, Zhu CH, Tian JH, Zhang LJ, Zhu H et al: F-fluorodeoxyglucose positron emission tomography/computed tomography-based radiomic features for prediction of epidermal growth factor receptor mutation status and prognosis in patients with lung adenocarcinoma. Transl Lung Cancer Res 2020, 9(3):563-574.

29. Carey KD, Garton AJ, Romero MS, Kahler J, Thomson S, Ross S, Park F, Haley JD, Gibson N, Sliwkowski MX: Kinetic analysis of epidermal growth factor receptor somatic mutant proteins shows increased sensitivity to the epidermal growth factor receptor tyrosine kinase inhibitor, erlotinib. Cancer Res 2006, 66(16):8163-8171.

\section{Figures}



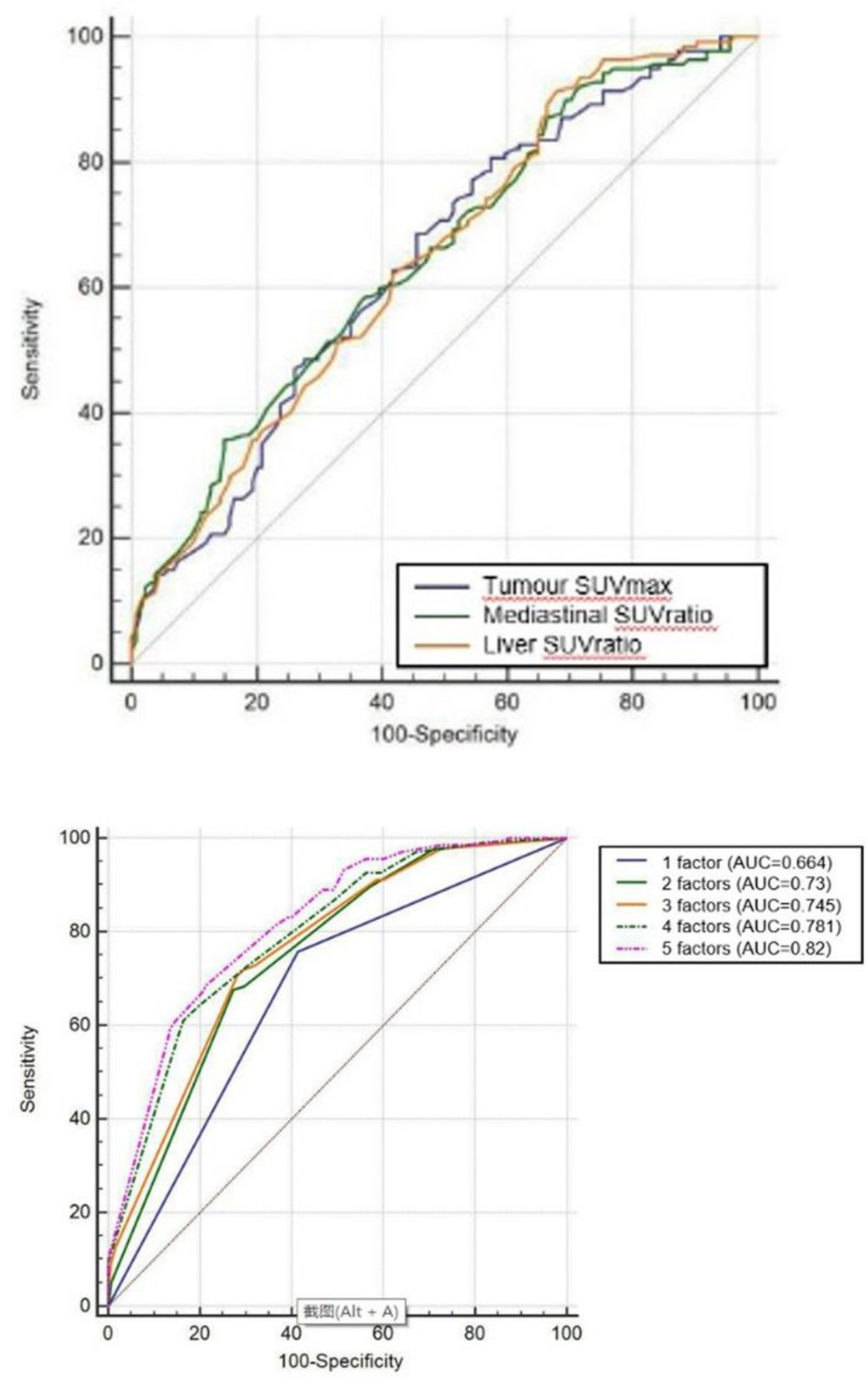

\section{Figure 1}

a: The ROC curve of PET/CT metabolic parameters for predicting EGFR mutations. b: ROC curves of different numbers of predictors. 